\title{
Caring for a child with type 1 diabetes: Links between family cohesion, perceived impact and parental adjustment
}

\author{
Helena Moreira ${ }^{1}$, Roberta Frontini ${ }^{1}$, Monika Bullinger $^{2}, \&$ Maria Cristina Canavarro ${ }^{1}$ \\ ${ }^{1}$ Faculty of Psychology and Education Sciences, University of Coimbra, Coimbra, Portugal \\ ${ }^{2}$ Institute of Medical Psychology, University Medical Center, Hamburg-Eppendorf, Hamburg, Germany
}

Cite as:

Moreira, H., Frontini, R., Bullinger, M. \& Canavarro, M. C. (2013). Caring for a child with type 1 diabetes: Links between family cohesion, perceived impact and parental adjustment. Journal of Family Psychology, 27(5), 731-742. doi:10.1037/a0034198.

URL: http://psycnet.apa.org/index.cfm?fa=buy.optionToBuy\&id=2013-31570-001

\section{Corresponding author:}

Helena Moreira (Moreira, H.)

Faculty of Psychology and Educational Sciences, University of Coimbra, Portugal

Faculdade de Psicologia e Ciências da Educação, Universidade de Coimbra, Rua do Colégio Novo, Apartado 6153, 3001-802 Coimbra, Portugal.

Telephone: 00351239851450

E-mail: hmoreira@fpce.uc.pt 


\begin{abstract}
This study analyzed the psychological adjustment of parents of children and adolescents with T1D and examined whether family cohesion and parental adjustment were connected through the perceived impact of this condition on family functioning, as well as whether these links vary according to the child's age. In total, 104 parents of children (8-12 years old) and adolescents (13-18 years old) with T1D and 142 parents of healthy children and adolescents completed self-report measures of family cohesion, parental stress, quality of life, anxious and depressive symptoms, and perceived impact of chronic illness. The results indicated that the parents of children and adolescents with T1D were more anxious and perceived less family cohesion than the parents of healthy children and adolescents. No significant differences were found on depressive symptoms, quality of life and parental stress. The hypothesized moderated mediation model showed that parents who perceived their family environments as more supportive and caring tended to evaluate the impact of T1D on their families less negatively; in turn, this perception was associated with better quality of life and less parental stress and anxious and depressive symptoms. These associations were independent of the children's age. Based on a risk and resistance framework, this study highlighted the relevance of the perceived impact of T1D on the family as a mechanism explaining the link between cohesion and parental adjustment. Familycentered interventions that help parents better cope with the demands of T1D and help family members be more supportive of each other seem to be particularly useful in this context.
\end{abstract}

Keywords: pediatric diabetes; family cohesion; perceived impact; parental adjustment. 
A childhood chronic condition, such as type 1 diabetes (T1D), impacts and is affected by the whole family (Drotar, 1997; Kazak, 1997; Wallander et al., 1989; Wallander \& Varni, 1998). It affects not only the welfare of the child but also the psychological adjustment of the parents. Indeed, the parents of children and adolescents (for brevity, the term children will be used henceforth to describe both children and adolescents) with T1D are exposed to considerable levels of stress, as they have to maintain their regular parenting roles while caring for a child with a chronic condition that requires adherence to an intensive medical regimen (Streisand, Braniecki, Tercyak, \& Kazak, 2001; Streisand, Mackey, \& Herge, 2010). Usually, parents take considerable responsibility for the treatment management, particularly during the pre-adolescent years, which includes several tasks such as the monitoring of blood glucose levels and insulin administration several times a day, as well as a heightened attention to physical activity and dietary intake (Drotar, 2006; Wysocki, Buckloh, \& Greco, 2009). Consequently, family functioning may be disturbed (Overstreet et al., 1995), and the likelihood of parental maladjustment may increase (De Beaufort \& Barnard, 2012). Despite this increased risk, research has focused on the child's adjustment, with parental experiences receiving much less attention. For instance, although the influence of family functioning on children's health-related outcomes, such as glycemic control, is well established (Cohen, Lumley, Naar-King, Partridge, \& Cakan, 2004), little is known about its impact on parental adjustment or the mechanisms that may account for the links between these variables. The present study examined the psychological adjustment of parents based on their anxious and depressive symptoms, parental stress and quality of life (QoL). Based on the disability-stresscoping model (Wallander \& Varni, 1992, 1998), this study also explored a moderatedmediation hypothesis, examining whether family cohesion was associated with parental adjustment through parents' perceptions of the impact of T1D on family functioning and whether these links vary according to the child's age. 


\section{Parental Psychological Adjustment}

Research has shown that the parents of children with T1D, particularly the mothers, have an increased risk of developing depressive symptoms (Driscoll et al., 2010; Jaser, Whittemore, Ambrosino, Lindemann, \& Grey, 2008; Kovaks, Obrosky, Goldston, \& Bonar, 1997; Streisand et al., 2008; Williams, Laffel, \& Hood, 2009), anxious symptoms (Horsh, McManus, Kennedy, \& Edge, 2007; Streisand et al., 2008; Williams et al., 2009) and even posttraumatic stress disorder (Horsh et al., 2007). The prevalence rates of clinically significant levels of depressive symptoms vary across studies and the measures used. For instance, when using the CES-D, the prevalence rates vary between 22.2\% (Jaser et al., 2008) and 33.3\% (Driscoll et al., 2010), although higher values (74\%) were found among the parents of newly diagnosed children (Streisand et al., 2008). Using the Hospital Anxiety and Depression Scale, Horsch et al. (2007) found that approximately $17 \%$ of parents reported moderate to severe symptoms of depression. The prevalence rates of anxiety range from 43.3\% (Horsch et al., 2007) to 59\% (Streisand et al., 2008). In addition, it has been found that parents of chronically ill children are likely to experience elevated levels of parental stress, or the stress related to caregiving and parenting tasks (Mullins et al., 2004; Streisand, Swift, Wickmark, Chen, \& Holmes, 2005).

Currently, it is widely accepted that despite the greater likelihood of parental psychological difficulties, there is considerable variation in the parents' adjustment (Wallander et al., 1989; Wallander \& Varni, 1998). Not every parent reports clinically significant psychological problems, and the majority show resilience in the face of these stressful events (Wallander \& Varni, 1998). To account for this variability, Wallander and Varni (1992; 1998; Wallander et al., 1989) proposed the disability-stress-coping model, which has been extensively used as a conceptual framework to examine the adjustment of disabled or chronically ill children and their parents (Lustig, Ireys, Sills, \& Walsh, 1996; 
Manuel, 2001; Mullins, Olson, Reyes, Bernardy, Huszti, \& Volk, 1991; Wallander et al., 1989). It proposes that adjustment to a chronic illness cannot be entirely explained by illness severity and that modifiable risk and resistance or protective factors should be considered to fully understand adjustment processes and their diversity. This model also hypothesizes that the associations between risk factors (including disease/disability parameters, functional care strain, and psychosocial stressors) and child and parental adjustment are moderated by resistance factors (including intrapersonal, social-ecological and stress processing factors). Intrapersonal factors refer to stable person factors, such as sense of challenge or perceived mastery, while social-ecological factors include aspects such as family environment, family resources or social support; stress processing, on the other hand, refers to the cognitive appraisal of the impact of the chronic illness on family and to coping strategies. Risk and resistance factors may have direct or indirect effects on adjustment. For instance, intrapersonal and social-ecological factors may be linked with adjustment in a direct or indirect way through stress processing factors. Given its complexity, only specific components of this theoretical model have been tested over the years (Wallander et al., 1989; Wallander \& Varni, 1998).

In this study, we focused on the association between two resistance factors and parents' adjustment. Specifically, we aimed to examine if the link between the perceived cohesion of family environment (social-ecological factor) and parents' adjustment is mediated by the cognitive appraisal of the impact of T1D (stress processing factor). It is fundamental to move beyond descriptions of relationships between variables to the elucidation of the mechanisms or processes underlying these relationships. Although this need has been emphasized by Wallander and Varni (1998), few studies have been conducted so far that attempted to clarify possible mechanisms linking family and individual variables in pediatric populations and, specifically, in children with T1D and their parents. 
The Indirect Effect of Family Cohesion on Parental Adjustment through Parents' Perceptions of the Impact of T1D on Family Functioning

One of the most frequently investigated social-ecological factors is the family environment (Wallander, Thompson, \& Alriksson-Schmidt, 1995), which is often conceptualized in terms of cohesion, conflict, and expressiveness (Field \& Duchoslav, 2009). Some studies have shown that the family environment has a strong influence on several physical and psychosocial outcomes of children, such as glycemic control and adherence (Cohen et al., 2004), health-related quality of life (Weissberg-Benchell et al., 2009) and externalizing (Hamlett, Pellegrini, \& Katz, 1992) and internalizing behaviors (Missotten, Luyckx, \& Seiffge-Krenke, 2012). Much less investigated has been its role on parents' adjustment. Wallander et al. (1989) found that in a sample of children with spina bifida and cerebral palsy, as well as their parents, a more positive family environment, as assessed by cohesion, expressiveness, and conflict, was correlated with better maternal adjustment. Similarly, Blankfeld and Holahan (1996) found that higher levels of family support (assessed by cohesion, conflict and expressiveness) predicted fewer depressive symptoms among mothers of children with diabetes and that this association was mediated by their adaptive coping.

In the present study, we focus on family cohesion, which is considered a key dimension of family environment (Holmbeck, Coakley, Hommeyer, Shapera, \& Westhoven, 2002; Missotten et al., 2012). Cohesive families are characterized by supportive, appropriate, healthy and positive interactions among family members (Field \& Duchoslav, 2009; Soliday, Kool, \& Lande, 2001). A few studies have examined the impact of T1D on family cohesion by comparing families of children with this chronic condition and families with healthy children. The results are not consistent, with some studies showing that families of children with T1D have lower levels of cohesion (Overstreet et al., 1995) and others reporting no 
differences between the groups (Hamlett et al., 1992). Furthermore, higher levels of family cohesion have been associated with better glycemic control, adherence and psychosocial functioning of children (Cohen et al., 2004). However, knowledge about the specific role of this construct in the adjustment of parents in the context of this chronic condition is still extremely limited.

Consistent with Wallander and Varni's model (1992, 1998; Wallander et al., 1989), the influence of family cohesion on parental adjustment is either direct or mediated by their cognitive appraisals (i.e., by their perceptions of the impact of the child's illness on family functioning). Based on stress and coping models (Lazarus \& Folkman, 1984), cognitive appraisals and coping processes are considered to be key elements in the prediction of parental adjustment (Wallander et al., 1989; Wallander \& Varni, 1998). Ireys and Silver (1996) found that among families of children with various chronic illnesses, the maternal appraisal of the impact of the illness on the family mediated the link between condition parameters, or service use, and maternal mental health. Lustig et al. (1996) examined the effects of condition parameters on the mental health of mothers of children with juvenile rheumatoid arthritis and found that the effect of the child's functional status and medication type on maternal mental health was mediated by the maternal appraisal of the impact of the condition on the family. For the same chronic condition, Manuel (2001) found that maternal appraisals moderated the link between their illness stress and psychological symptoms. To our knowledge, no study has examined the role of cognitive appraisals among parents of children with T1D or their mediating effects on the association between family cohesion and parents' adjustment.

\section{The Current Study}

The present study had three goals. First, we aimed to investigate the psychological adjustment of the parents of children (between the ages of 8 and 12) and adolescents 
(between the ages of 13 and 18) with T1D by comparing their QoL, parental stress, and anxious and depressive symptoms with a comparison group of parents of healthy children and adolescents. We hypothesized that parents of children and adolescents with T1D would report higher levels of parental stress and psychopathological symptoms and a lower QoL than parents of healthy children and adolescents. We also intended to explore the role of the child's age on parental adjustment by analyzing potential differences between two developmental stages (childhood and adolescence). We hypothesized that parents of adolescents with T1D would report lower levels of psychological adjustment than parents of children with T1D and healthy children.

Second, we intended to compare the levels of family cohesion among parents of children and adolescents with T1D and parents of healthy children and adolescents, while taking into account the children's age. Lower levels of perceived cohesion are expected in the clinical group, particularly among parents of adolescents.

Third, within a risk and resistance framework (Wallander et al., 1989; Wallander \& Varni, 1992, 1998), we aimed to explore the indirect effect of family cohesion on parents' adjustment (QoL, parental stress, anxious and depressive symptoms) through their perceptions of the impact of T1D on family life. The specific focus on these factors is consistent with Wallander and Varni's research strategy (1998) of investigating only a few components or subparts of the global theoretical model in each study (e.g., Wallander et al., 1989). Because each study focuses on one specific component or a few specific components, some factors can be assumed to play different roles in different studies. Although socialecological factors are conceptualized as resistance factors (thus acting as moderators in the global model), family cohesion functions as an independent variable because of the narrower focus of the current study. Similarly, in previous studies examining the associations between family factors and parental adjustment (Blankfeld \& Holahan, 1996; Mullins et al., 1991; 
Wallander et al., 1989), family factors or other social-ecological factors have been independent variables. The perception of the impact of T1D on family life was conceptualized as a mediator, in accordance with previous studies (Ireys \& Silver, 1996; Lustig et al., 1996) and with the theoretical model in which interpersonal and socialecological factors can influence adjustment indirectly through individual cognitive appraisal. In the present study, we hypothesized that the perceived impact would mediate the association between family cohesion and parental adjustment. Specifically, we expected that higher levels of cohesion would be associated with a less negative appraisal of the impact of T1D on family and that less negative appraisals would be associated with better adjustment.

In addition, we intended to explore whether the proposed mediation model is moderated by the child's age. Although the disability-stress-coping model does not explicitly consider this factor, Wallander and Varni (1998) acknowledged the need to adopt a developmental perspective in the theory and research addressing individual and family adjustments to pediatric chronic conditions. Accordingly, they emphasized that children and their families are in continuous development (Wallander \& Varni, 1998; Wallander et al., 1995) and that it is imperative that the research reflects this aspect.

\section{Methods}

\section{Participants}

The participants included 104 parents of children with T1D (Group 1 - G1) and 142 parents of healthy children (Group $2-\mathrm{G} 2$ ), aged 8 to 18 years. To be included in this study, the G1 parents had to be the primary caregivers (i.e., must identify themselves as primarily responsible for their child's diabetes management), and their children had to be aged between 8 and 18 years at the time of recruitment, had to be diagnosed with T1D for at least 6 months, and had no serious comorbid medical or mental conditions. The G2 parents had to have a healthy child with no diagnosis of a chronic health condition, aged between 8 and 18 years at 
the time of recruitment. The parents of children with significant developmental delays or severe psychiatric disorders were excluded. The parents' socio-demographic characteristics and the clinical characteristics of children with T1D are presented in Table 1. As presented in Table 1, no significant differences between the two groups were found.

\section{Procedure}

G1 parents were recruited through the pediatric departments of two Portuguese public and urban hospitals in the central region of Portugal between February and September of 2012. The Ethics Committee and Direction Boards of both hospitals approved the study. The participants completed the self-report questionnaires and the informed consent in a consultation office provided for this purpose, either before or after their children's diabetes medical appointments. Trained research assistants were available to assist them whenever necessary. G2 parents were collected through a Portuguese public school after the Direction Board had authorized the study. The parents were given letters explaining the study and the informed consent form. Those who agreed to participate in the study and returned the informed consent form received packets with questionnaires to complete at home and return a week later. Insert Table 1 about here

\section{Measures}

Family cohesion. To assess family cohesion, the Cohesion subscale of the Relationship dimension of the Portuguese version of the Family Environment Scale (FES; Moos \& Moos, 1986; Matos \& Fontaine, 1992) was used. The FES has been widely used with families of children with various chronic health conditions, including diabetes (e.g., Holmbeck et al., 2002; Overstreet et al., 1995), and is considered an appropriate and useful measure of family functioning in pediatric psychology research (Alderfer et al., 2008). The Cohesion subscale assesses an individual's perceptions of commitment to the family and the degree to which family members are helpful and supportive of one another. It is composed of 
nine items (e.g., "Family members really help and support one another"), answered on a 6point Likert scale ranging from 1 (completely disagree) to 6 (completely agree). Higher scores indicate greater perceptions of cohesion within the family environment. The Cronbach's alphas were .85 (G1) and $.82(\mathrm{G} 2)$.

Psychopathological symptoms. The Portuguese version of the Hospital Anxiety and Depression Scale (HADS; Pais-Ribeiro et al., 2007; Zigmond \& Snaith, 1983) was used to assess current levels of depressive (e.g. "I have lost interest in my appearance") and anxious (e.g. "I feel tense or 'wound up'") symptomatology. This scale contains 14 items and uses a 4point scale that ranges from 0 (not at all/ only occasionally) to 3 (most of the time/ a great deal of the time), with higher scores indicating higher levels of symptomatology. Scores between 0 and 7 are considered "normal", between 8 and 10 "mild", between 11 and 14 "'moderate"' and between 15 and 21 "severe"'. According to Snaith (2003), scores of 11 or higher indicate the possible presence ("caseness") of a mood disorder. In this sample, the Cronbach's alpha coefficients were .81 (G1) and .77 (G2) for anxiety and .76 (G1) and .70 (G2) for depression.

Parental stress. To assess the distress associated with the parental role, the Parental Distress subscale of the Portuguese version of the Parenting Stress Index - Short Form (PSISF; Abidin, 1995) was used. This subscale has 12 items that are answered on a 5-point Likert scale ranging from 1 (completely disagree) to 5 (completely agree). It assesses several aspects related to the distress of parenting, such as life restrictions due to the demands of child-rearing ("I feel trapped by my responsibilities as a parent"). Higher scores indicate higher levels of parental stress. The PSI-SF is one of the most commonly used measures of parenting stress. Although it is intended for parents of children 12 years and younger, it has been considered appropriate for use with both children and adolescents (e.g., Radcliff, 
Bennett, Kazak, Foley, \& Phillips, 1996; Schaaijk, Roeleveld-Versteegh, \& Baar, 2013; Streisand et al., 2001). The Cronbach's alphas were .88 (G1) and .83 (G2).

Quality of life. To assess the parents' perceptions of their QoL, the Portuguese version of the EUROHIS-QOL 8-item index (Pereira, Melo, Gameiro \& Canavarro, 2011; Schmidt, Mühlan, \& Power, 2006) was used. This instrument is a brief indicator of overall QoL and is derived from the World Health Organization Quality of Life (WHOQOL) project (WHOQOL Group, 1995), an international collaboration that has developed internationally applicable and cross-culturally comparable QoL assessment instruments (e.g., WHOQOL100 and WHOQOL-bref). The EUROHIS-QOL 8-item index was developed as an adaptation of the WHOQOL instruments, and it contains two items per WHOQOL domain (social, psychological, physical and environmental). The overall QoL score is the sum of the eight items (e.g., "How satisfied are you with your health?", "Have you enough energy for everyday life?"). It uses a 5-point Likert scale ranging from 1 (not at all/very dissatisfied) to 5 (completely/very satisfied), with a higher score indicating a better QoL. In the present study, Cronbach's alphas were $.82(\mathrm{G} 1)$ and $.85(\mathrm{G} 2)$.

Perceived impact of pediatric chronic illness. To measure the parents' appraisals of the negative impact of T1D on the family, the Portuguese version of the Impact on Family Scale - Revised (IOF-R; Stein \& Jessop, 2003; Albuquerque, Fonseca, Pereira, Nazaré, \& Canavarro, 2011) was used. The IOF-R is considered a "well-established" measure of the negative impact of a child's chronic illness on the family (Alderfer et al., 2008) because of its excellent psychometric properties and appropriateness in pediatric contexts. As in the present study, this instrument has been widely used to assess the cognitive appraisal component of Wallander and Varni's model (e.g., Manuel, 1992; Lustig et al., 1996). The IOF-R has a unidimensional structure and consists of 15 items answered on a 5-point Likert scale ranging 
from 1 (strongly disagree) to 5 (strongly agree). A higher score indicates a greater negative impact of chronic illness. Cronbach's alpha coefficient was .90 (G1).

Sociodemographic and clinical information. The sociodemographic data included the children's age and gender as well as the parents' age, gender, marital status, professional status, family income, and education. The clinical data were self-reported by parents and included the durations of T1D, the insulin regimens, the number of blood glucose checks per day, the frequency of hypoglycemia episodes and the level of metabolic control. The frequency of hypoglycemia episodes in the last three months was assessed using a single item (“How frequent are episodes of hypoglycemia?”) answered on a 4-point Likert scale ranging from 1 (not at all frequent) to 4 (very frequent). The level of metabolic control was assessed using the glycosylated hemoglobin (HbAlc) level, which represents the average blood glucose during the past 2-3 months. Higher levels of HbAlc indicate poorer metabolic control.

\section{Data Analyses}

The data analyses were conducted using the Statistical Package for the Social Sciences (SPSS, version 20.0; IBM SPSS, Chicago, IL). Any missing data for the study variables occurred randomly and at a low frequency $(<5 \%)$ and were handled by listwise deletion. Descriptive statistics were computed for all socio-demographic, clinical and study variables. The potential differences between G1 and G2 for the socio-demographic variables were analyzed using chi-squared tests (age group, children and parents' gender, marital status, education, monthly income and professional status) and one-way ANOVAs (children's and parents' age).

Two-way ANOVAs, using the group (G1, G2) and age categories (children, adolescents) as independent variables, were performed for QoL, parental stress and family cohesion. A two-way MANOVA, also using the group and age categories as independent 
variables, was performed for anxious and depressive symptoms. The Pillai's trace tested the significance of the multivariate main effects and interactions; it was chosen because of its robustness, which is particularly important with unequal sample sizes in each group (Tabachnick \& Fidell, 2007). When a multivariate effect was found, subsequent two-way ANOVAs were performed for each dependent variable. Finally, because the perceived impact was only assessed among the G1 parents, a one-way ANOVA was performed to assess the differences between parents of children and parents of adolescents.

The Process computation tool, a SPSS macro for path analysis-based moderation and mediation analyses, as well as their combination in the so-called "conditional process analysis" (Hayes, 2013), was used to estimate moderated mediation and simple mediation models. Initially, four moderated mediation analyses were performed to examine whether the direct and indirect effects of family cohesion (independent variable; IV) on indicators of parental adjustment (dependent variables; DV), through parental appraisals of the family impact of T1D (mediator; M), varied according to the two categories of the child's age group (moderator). In the current study, the moderator was hypothesized to affect the path linking the IV and the M (path $a$ ), the path linking the M and the DV (path $b$ ), and the direct effect (path c'). This model correspond to the "model 59" in Hayes (2013) or the "total effect moderation model" in Edwards and Lambert (2007). Therefore, three interactions were tested in each model (cohesion $\mathrm{x}$ age group in paths $a$ and $c^{\prime}$; perceived impact $\mathrm{x}$ age group in path $b$ ). Prior to model estimation, the variables used in the construction of products were meancentered (Aiken \& West, 1991).

In the absence of a significant interaction in one or more paths, the models were reestimated after the removal of nonsignificant interactions. This resulted in the estimation of simple mediation models ("model 4" in Hayes (2013) or "basic mediator model" in Edwards and Lambert (2007)) in all the dependent variables. A bootstrapping procedure was used to 
assess unconditional indirect effects (using 5000 resamples). Bootstrapping is a nonparametric resampling procedure that is recommended for testing indirect effects because it does not require the assumption of normality of the sampling distribution of the indirect effects (Hayes, 2009). This procedure creates 95\% bias-corrected and accelerated confidence intervals (BCa CIs) of the indirect effects, with an indirect effect considered to be significantly different from zero if zero is not contained within the lower and upper CIs.

Significance was set at the .05 level, and partial eta squared $\left(\eta_{p}^{2}\right)$ provided the estimate of the effect size for the analyses of variance. Cohen's (1988) guidelines were used for describing the effect sizes of reported correlations (i.e., small for correlations around .10, medium for those near .30 , and large for correlations at .50 or higher). Post hoc power calculations (G*Power; Faul, Erdfelder, Lang, \& Buchner, 2007) performed for analyses of variance, with a significance level of .05 and power of .80 , demonstrated that medium to large effects could be detected.

\section{Results}

\section{Group Differences and Correlations Among Study Variables}

Regarding the severity of anxious symptoms, a significant difference was found between $\mathrm{G} 1$ and $\mathrm{G} 2, \chi^{2}(3, N=246)=18.97, p<.000$. As presented in Table $1,31(29.8 \%)$ parents of children with T1D scored above 11 (moderate and severe categories), indicating the probable presence of an anxiety disorder. In regard to the depressive symptoms, no significant differences were found between groups, $\chi^{2}(3, N=246)=4.04, p=.26$. Only 11 (10.6\%) parents of children with T1D scored above 11, indicating the probable presence of a mood disorder.

Table 2 presents the descriptive statistics of study variables according to group (G1, G2) and age categories (children, adolescents), as well as the group, age and interaction effects. With regard to the parents' perceptions of family cohesion a significant group effect 
was found, $F(1,242)=10.84, p=.001, \eta_{\mathrm{p}}^{2}=.043$, with the parents of children and adolescents with T1D reporting less cohesion than the parents of healthy children and adolescents. No differences were found between the parents of children and the parents of adolescents, $F(1,242)=1.81, p=.179, \eta_{\mathrm{p}}^{2}=.007$, and no interaction was found between the group and age categories, $F(1,242)=1.66, p=.199, \eta_{\mathrm{p}}^{2}=.007$. Regarding parents' psychopathological symptoms, a significant multivariate group effect was found, Pillai's trace $=0.04, F(2,241)=5.30, p=.006, \eta_{p}^{2}=.042$. The subsequent univariate analyses revealed a significant difference between G1 and G2 for anxious symptomatology, $F(1,242)$ $=10.21, p=.002, \eta_{\mathrm{p}}^{2}=.040$, with G1 parents reporting more anxiety than G2 ones. No difference was found for depressive symptomatology, $F(1,242)=2.95, p=.087, \eta_{\mathrm{p}}^{2}=.012$. The multivariate age effect, Pillai's trace $=0.003, F(2,241)=0.40, p=.671, \eta_{p}^{2}=.003$, and the multivariate interaction effect, Pillai's trace $=0.009, F(2,241)=1.13, p=.326, \eta_{\mathrm{p}}^{2}=$ .009 , were not significant. With regard to parental stress, an age difference was found, $F(1$, $242)=6.45, p=.012, \eta_{\mathrm{p}}^{2}=.027$, with parents of adolescents presenting higher levels of parental stress than the parents of children. The group, $F(1,242)=2.23, p=.136, \eta_{\mathrm{p}}^{2}=.009$, and the interaction, $F(1,242)=0.01, p=.944, \eta_{p}^{2}=.000$, effects were not significant. For QoL, a marginally significant difference was found between groups, $F(1,242)=3.59$, $p=$ $.059, \eta_{\mathrm{p}}^{2}=.015$. No significant differences were found between age categories, $F(1,242)=$ $0.11, p=.741, \eta_{\mathrm{p}}^{2}=.000$, and the interaction was not significant, $F(1,242)=0.12, p=.728$ $\eta_{\mathrm{p}}^{2}=.001$. Finally, the perceived impact of T1D on families was only assessed among G1 parents. A significant difference was found between the parents of children and the parents of adolescents, $F(1,102)=10.01, p=.002, \eta_{\mathrm{p}}^{2}=.092$, with the former reporting a more negative impact of the illness.

Medium to high correlations were found among the study variables in both groups of parents. Family cohesion was positively correlated with QoL and negatively correlated with 
anxiety, depression and parental stress. Among the G1 parents, higher levels of the negative impact of T1D were correlated with higher levels of depression, anxiety, and parental stress and with lower cohesion and QoL. Insert Table 2 about here

\section{Moderated Mediation Analyses}

To examine whether the indirect effect of family cohesion on parental adjustment through parental perception of the impact of T1D on family varied across the two age groups $(0=$ children, $1=$ adolescents $)$, four moderated mediation models were estimated, one for each DV (parental stress, QoL, anxious and depressive symptomatology). In each model, the parents' perception of family cohesion was the IV, the perceived impact of T1D was the M, and the children's age group was the moderator.

These analyses revealed that both the paths from the cohesion to the perceived impact (path $a$ ) and from the perceived impact to each indicator of parental adjustment (path $b$ ) were not moderated by the child's age. Specifically, no statistically significant interactions were found between children's age group and the family cohesion $(b=1.91, S E=1.60, p=.24)$ and between the children's age group and the perceived impact of T1D in each of the four estimated models (QoL: $b=-0.50, S E=0.40, p=.23$; parental stress: $b=-0.42, S E=0.22, p$ $=.07$; depressive symptoms: $b=-0.12, S E=0.10, p=.24$; anxious symptoms: $b=-0.01, S E$ $=0.11, p=.90)$, suggesting that the children's age group does not moderate the indirect effect of cohesion on parental adjustment. With regard to the direct effect of family cohesion on the dependent variables, no significant interactions were found between children's age group and the family cohesion in the models of QoL $(b=-6.09, S E=3.70, p=.10)$, parental stress $(b=$ $-3.34, S E=2.00, p=.10)$, depressive symptoms $(b=-0.39, S E=0.90, p=.67)$, and anxious symptoms $(b=0.11, S E=1.05, p=.91)$. Because no significant interactions were found, the conditional indirect effects were not analyzed and simple mediation models (without the children's age group as the moderator) were estimated. 


\section{Mediation Analyses}

Four simple mediation models were tested. The effects of the IV on the proposed M (path $a$ ), the effects of M on the DV partialling out the effect of IV (path $b$ ), the direct effect of IV on DV after controlling for M (path $c^{\prime}$ ) and the total effect of IV on DV (path $c$ ) are presented in Table 3. Significant indirect effects of IV on DV through M (a*b) were found for QoL (point estimate $=1.24,95 \% \mathrm{BCaCI}=0.06 / 2.99)$, parental stress $($ point estimate $=$ $1.78,95 \% \mathrm{BCaCI}=-3.13 /-0.88$ ), anxious symptoms (point estimate $=-0.65,95 \% \mathrm{BCaCI}=-$ $1.30 /-0.26$ ), and depressive symptoms (point estimate $=-0.76,95 \% \mathrm{BCaCI}=-1.40 /-0.38$ ). These results indicate that higher levels of family cohesion were associated with a less negative impact from T1D, which, in turn, was associated with a better QoL and lower levels of parental stress and anxious and depressive symptoms. Insert Table 3 about here

\section{Discussion}

The present study sought to examine the psychological adjustment of parents of children with T1D and to explore a mediation model in which cohesion was associated with parental adjustment through the perceived impact of T1D on family functioning. Overall, the results showed that although parents of children with T1D reported lower cohesion and higher anxiety, their levels of parental stress, QoL and depressive symptoms were similar to those of the parents of healthy children. Moreover, it was found that the parents' cognitive appraisals of the impact of T1D are a possible mechanism explaining the link between cohesion and several indicators of parental adjustment. Interestingly, these associations were independent of the child's age.

As expected, higher levels of anxiety were found among parents of children with T1D compared to parents of healthy children, regardless of children's age. Additionally, $29.8 \%$ of parents scored above 11 on the anxiety subscale of the HADS, indicating the probable presence of an anxiety disorder. These findings are in accordance with the literature, which 
has consistently shown that parents of chronically ill children, specifically those diagnosed with T1D, are at greater risk of developing psychopathological symptoms or disorders (Driscoll et al., 2010; Jaser et al., 2008; Kovaks et al., 1997). For instance, Horsch et al. (2007), using the same measure, found that $43.3 \%$ of parents experienced clinical levels of anxious symptoms. This may be related to an increased burden resulting from caregiving and health-related tasks. Caring for a child with this chronic condition may be highly distressing for parents, as they are usually the main caregivers and take responsibility for the T1D treatment management, particularly during pre-adolescent years. Furthermore, parents may frequently worry about their children's futures, long-term medical complications, financial issues and time demands, as well as issues of illness management at school or when the child is in the care of another person, which expectedly causes parents to experience heightened levels of anxiety.

However, contrary to our expectations, no group differences were found for depressive symptoms and a small percentage (10.6\%) of participants scored above 11 on the HADS. In contrast with previous studies that reported elevated levels of depressive symptoms (Horsch et al., 2007; Jaser et al., 2008), our sample was quite heterogeneous with regard the duration of T1D, and included children that had been diagnosed with T1D for a long time. These differences may help explain our results, since parents of children whose duration of T1D was higher may have developed, over time, more effective strategies to cope with the demands of the disease, therefore presenting lower levels of depressive symptoms. Moreover, contrary to other studies, such as the study of Horsch et al. (2007) that included parents of young children (1-16 years), our sample included parents of children aged 8 to 18 years. The caregiving demands of young children are likely to be more demanding because of their greater dependency, which may have contributed to the higher levels of depressive symptoms in that study population. In addition, these results may be related to the fact that 
depression is more tightly associated with a perception or experience of loss or selfdevaluation, whereas anxiety is more connected to an experience or perception of threat or danger (Beck \& Emery, 1985). In fact, T1D is characterized by a certain degree of unpredictability. For instance, episodes of hypoglycemia (extremely low blood glucose levels) may suddenly occur and, if untreated, may result in severe complications. Some studies have reported that many parents experience significant and persistent fear of hypoglycemia (Bernard, Thomas, Royle, Noyes, \& Waugh, 2010; Streisand et al., 2005). This unpredictability, together with the complexity of the treatment regimen, can contribute to high levels of parental anxiety but not so much to increased levels of depressive symptoms. Furthermore, we should not exclude the hypothesis that the absence of a significant difference between groups might be due to a low statistical power to detect small effect sizes, which might have been the case in this analysis. Post hoc power analysis showed that the sample size only allowed the detection of medium to large effects, meaning that this study could not detect any existing differences between groups that had small effect sizes.

Contrary to our expectations, no group differences were found for parental stress and the group effect for QoL was marginally significant. Nevertheless, considering the medium sample size and the presence of a marginally significant difference in QoL, it is desirable for future studies to increase the number of participants to ascertain whether an increment in statistical power would allow the emergence of significant differences. Additionally, a more comprehensive QoL measure (e.g. WHOQOL-bref) could be used to allow a more thorough analysis of this construct. Although psychometrically robust and highly recommended for the research context (Schmidt et al., 2006), EUROHIS-QOL-8 is a brief indicator of overall QoL and might not be able to detect particular issues related to caring for a child with a chronic condition (e.g. role changes, illness management responsibility). It is a generic QoL instrument, which compared to a condition-specific measure, may not be as sensitive to 
differences associated with a specific child's condition or treatment (Chow, Morrow, Robbins, \& Leask, 2013), although it has the important advantage of allowing comparison between groups. Similarly, although the PSI-SF has been widely used to assess parental stress in families of chronically ill children and adolescents (e.g., Radcliff et al., 1996; Schaaijk et al., 2013), a more specific instrument (e.g., Pediatric Inventory for Parents; Streisand et al., 2001) could have been more sensitive to the specific challenges and demands that parents of children with T1D confront.

Contrary to our initial hypothesis, no age differences within the clinical group were found for QoL, anxiety or depression, which suggests that caring for a child or an adolescent with T1D may be equally challenging. Nevertheless, an age effect was detected for both parental stress and perceived impact of illness. With regards to parental stress, we found that parents of adolescents (aged between 13 and 18) reported significantly more parental stress than the parents of children. This difference, which is independent of the type of group (G1 or G2), is most likely associated with the normative changes that characterize the transition to adolescence, when youths become more autonomous and independent from their parents (Drew, Berg, \& Wiebe, 2010). This is a developmental period marked by important challenges and transformations within the parent-youth dyad, and some level of parental stress might be expected (Small, Eastman, \& Cornelius, 1988). Moreover, we found that parents of children (aged 8 to 12) reported a more negative impact of T1D on their family lives than parents of adolescents. During infancy, children are very dependent upon their parents and, consequently, the impact of the illness on family life is most likely higher. Some aspects that might be negatively affected by T1D, and which are assessed by the Impact on Family Scale-Revised, such as finding a reliable person to take care of the child or the possibility of traveling out of town or being with family and friends, are not likely to be affected during adolescence. The adolescent demand for autonomy and the transference of 
responsibility for diabetes care from parents to adolescents that gradually occurs during the transition to adolescence (Anderson et al., 1997), lead to a lower impact of the illness and its management on the functioning of the family.

As expected, lower levels of family cohesion were found among the parents of children with T1D. However, contrary to our hypothesis, these associations were independent of the child's age. The intensive regimen treatment, the parents' responsibility for T1D management and even the shift in responsibility during the transition to adolescence may, to some extent, disrupt family functioning and relationships within the family system, decreasing the perceptions of proximity, connectedness and support within the family. Similar results were found in previous studies (Overstreet et al., 1995), although the literature is not consistent regarding this matter (Hamlett et al., 1992; Missotten et al., 2012).

Our findings elucidated a mechanism through which family cohesion and parents' adjustment are linked. Specifically, we found that parents who perceived their family environments as more supportive and caring tended to evaluate the impact of T1D on their family lives less negatively and to show better adjustment. When family members are supportive of each other, the strains imposed by T1D are more likely to be shared, which appears to be associated with less parental depression, anxiety and parental stress and more positive evaluations of QoL. As expected, these relationships were explained by the parents' cognitive appraisals (i.e., by their perceptions of the impact of T1D on their families and social lives). Supportive family relationships may allow parents to feel less burdened by the demands of T1D, resulting in their assessing the disease's impact on family and social life in less negative ways. In addition, when parents evaluate their children's chronic conditions as less burdening and less demanding, they are more likely to show better adjustment. This mediation hypothesis is in accordance with the disability-stress-coping model (Wallander \& Varni, 1992, 1998), which proposes that risk and resistance factors may be associated with 
parents' adjustments via their cognitive appraisals. The way parents perceive the restrictions in their lives that are imposed by the illness (e.g., seeing family and friends less frequently, feeling little desire to go out, thinking about not having more children, not being able to travel out of town) seems to explain the associations with cohesion and adjustment. To the best of our knowledge, no previous studies have examined these links among parents of children with T1D.

We initially proposed a moderated mediation model, with the children's age group as the moderator, to explore the role of the developmental stage of the child in the associations between cohesion, perceived impact and parental adjustment. However, we found that the mediation models were not moderated by the child's age, suggesting that the associations amongst these variables occur in both parents of children and adolescents.

\section{Limitations and Strengths}

The cross-sectional design is the main limitation of this study. Adjustment to a chronic condition is an ongoing dynamic process, not a static outcome (Rentinck, Ketelaar, Jongman, \& Gorter, 2006), and it can be better understood through a longitudinal design. Furthermore, cross-sectional studies do not allow causal inferences to be made, meaning that the observed associations among variables can be bidirectional (e.g. family cohesion can predict or be predicted by better adjustment). Future longitudinal studies are warranted to explore the dynamic process of adjustment and to determine the direction of associations over time. Second, 90.4\% (G1) and 95.8\% (G2) of primary caregivers were mothers. Although this disproportion between the participation levels of mothers and fathers is common in pediatric research, and we intended to recruit primary caregivers regardless of gender, it would be relevant to increase the number of fathers and to assess role differences. Third, the reliance on the parents' self-reported data is another limitation of the current study. This chronic condition may be perceived and experienced by family members in different ways, 
and its impact on the individual adjustment of each member may be different. Multiple perspectives within the family are desirable (Alderfer et al., 2008), particularly when assessing aspects such as family cohesion and the perceived impact of T1D on the family. Future studies should include self-reports from all family members. Fourth, the representativeness of the sample cannot be guaranteed, as parents were recruited from a convenience sample at only two public hospitals in the central region of Portugal. Nevertheless, the sample's sociodemographic characteristics (family income, professional status and education) are similar to Portuguese national levels.

Despite these limitations, the current study demonstrated a number of strengths. Grounded on a solid theoretical framework (Wallander et al., 1989; Wallander \& Varni, 1992, 1998), this study tested a mediation model, whereby a social-ecological resistance factor was linked to parental adjustment through a stress processing resistance factor. To our knowledge this is the first study to investigate the association between these variables among parents of children with T1D. Another strength of the present study is the consideration of developmental differences between children and adolescents. Most studies analyze children and adolescents as a single group, ignoring any differences that may occur as a consequence of particular characteristics of each developmental stage. The use of an adequate comparison group, composed of parents of healthy children with equivalent sociodemographic characteristics, is another important aspect of this study. Finally, several adjustment indicators (QoL, parental stress and psychopathological symptoms) were analyzed, allowing a more complete picture of parents' adjustments to this chronic condition.

In conclusion, the present study provides a significant and innovative contribution to the understanding of the psychological adjustment of parents of children with T1D. Based on a risk and resistance framework (Wallander \& Varni, 1992, 1998), it highlights the relevance of the perceived impact of T1D on the family as a mechanism explaining the link between 
cohesion and parental adjustment. Our findings also have important clinical implications, as they show that the parents of children with T1D are at particularly high risk for higher levels of anxiety. Because parental adjustment has important implications for children's adjustment (Drotar, 1997), it is fundamental that health professionals assess parental mental health, including symptoms of anxiety and depression, as well as their QoL and intervene whenever necessary. Family-centered interventions that help parents cope better with the demands and tasks of illness and that promote and sustain family cohesion can be particularly useful. 


\section{References}

Aiken, L., \& West, S. (1991). Multiple regression: Testing and interpreting interactions. Thousand Oaks, CA: Sage Publications, Inc.

Abidin, R. R. (1995). Parenting Stress Index: Professional manual (3rd ed.). Odessa, FL: Psychological Assessment Resources, Inc.

Albuquerque, S., Fonseca, A., Pereira, M., Nazaré, B., \& Canavarro, M. C. (2011). Estudos psicométricos da versão Portuguesa da Escala de Impacto Familiar (EIF) [Psychometric studies of the Portuguese version of the Impact on Family Scale (IOF)]. Laboratório de Psicologia, 9, 175-189.

Alderfer, M. A., Fiese, B. H., Gold, J. I., Cutuli, J. J., Holmbeck, G. N., Goldbeck, L., ... Patterson, J. (2008). Evidenced-based assessment in pediatric psychology: Family measures. Journal of Pediatric Psychology, 33, 1046-1061.

Anderson, B., Ho, J., Brackett, J., Finkelstein, D., \& Laffel, L. (1997). Parental involvement in diabetes management tasks: Relationships to blood glucose monitoring adherence and metabolic control in young adolescents with insulin-dependent diabetes mellitus. The Journal of Pediatrics, 130, 257-265.

Beck, A. T., \& Emery, G. (1985). Anxiety disorders and phobias: A cognitive perspective. New York: Basic Books.

Bernard, K., Thomas, S., Royle, P., Noyes, K., \& Waugh, N. (2010). Fear of hypoglycemia in parents of young children with type 1 diabetes: A systematic review. BMC Pediatrics, 10, 50. doi: 10.1186/1471-2431-10-50.

Blankfeld, D. \& Holahan, C. (1996). Family support, coping strategies, and depressive symptoms among mothers of children with diabetes. Journal of Family Psychology, 10, 173-179. 
Cohen, J. (1988). Statistical power analysis for the behavioral sciences. Hillsdale, NJ: Erlbaum.

Cohen, D., Lumley, M., Naar-King, S., Partridge, T., \& Cakan, N. (2004). Child behavior problems and family functioning as predictors of adherence and glycemic control in economically disadvantaged children with Type 1 Diabetes: A prospective study. Journal of Pediatric Psychology, 29, 171-184. doi: 10.1093/jpepsy/jsh019

Chow, M. Y., Morrow, A. M., Robbins, S. C., \& Leask, J. (2013). Condition-specific quality of life questionnaires for caregivers of children with pediatric conditions: A systematic review. Quality of Life Research, Advance online publication. doi: 10.1007/s11136-0120343-Z

De Beaufort, C. \& Barnard, K. (2012). Challenges to emotional well-being: Depression, anxiety and parental fear of hypoglycemia. In D. Christie \& C. Martin (Eds.). Psychosocial aspects of diabetes: Children, adolescents and their families (pp. 38-52). London: Radcliffe Publishing.

Drew, L., Berg, C., \& Wiebe, D. (2010). The mediating role of extreme peer orientation in the relationships between adolescent-parent relationship and diabetes management. Journal of Family Psychology, 24, 299-306. doi: 10.1037/a0019352

Driscoll, K., Johnson, S., Barker, D., Quittner, A., Deeb, L., Geller, D., ... Silverstein, J. (2010). Risk factors associated with depressive symptoms in caregivers of children with type 1 diabetes or cystic fibrosis. Journal of Pediatric Psychology, 35, 814-822. doi: 10.1093/jpepsy/jsp138

Drotar, D. (1997). Relating parent and family functioning to the psychological adjustment of children with chronic health conditions: What have we learned? What do we need to know? Journal of Pediatric Psychology, 22, 149-165. doi: 10.1093/jpepsy/22.2.149 
Drotar (2006). Psychological interventions in childhood chronic illness. Washington, DC: American Psychological Association.

Edwards, J. R., \& Lambert, L. S. (2007). Methods for integrating moderation and mediation: A general analytical framework using moderated path analysis. Psychological Methods, 12, 1-22.

Faul, F., Erdfelder, E., Lang, A.-G., \& Buchner, A. (2007). G*Power 3: A flexible statistical power analysis program for the social, behavioural, and biomedical sciences. Behaviour Research Methods, 39, 175-191. doi:10.3758/BF03193146

Field, C. \& Duchoslav, R. (2009). Family influence on adolescent treatment outcomes. In W. T. O’Donohue \& L. W. Tolle (eds.), Behavioral approaches to chronic disease in adolescence (pp. 47-54). New York: Springer.

Hamlett, K., Pellegrini, D., \& Katz, K. (1992). Childhood chronic illness as a family stressor. Journal of Pediatric Psychology, 17, 33-47. doi: 10.1093/jpepsy/17.1.33

Hayes, A. F. (2009). Beyond Baron and Kenny: Statistical mediation analysis in the new millennium. Communication Monographs, 76, 408-420.

Hayes, A. F. (2013). Introduction to mediation, moderation, and conditional process analysis: A regression-based approach. New York: The Guilford Press.

Holmbeck, G., Coakley, R., Hommeyer, J., Shapera, W., \& Westhoven, V. (2002). Observed and perceived dyadic and systemic functioning in families of preadolescents with spina bifida. Journal of Pediatric Psychology, 27, 177-189. doi: 10.1093/jpepsy/27.2.177

Horsch, A., McManus, F., Kennedy, P., \& Edge, J. (2007). Anxiety, depressive, and posttraumatic stress symptoms in mothers of children with type 1 diabetes. Journal of Traumatic Stress, 20, 881-891. doi: 10.1002/jts.20247 
Ireys, H. Y. \& Silver, E. J. (1996). Perception of the impact of a child's chronic illness: Does it predict maternal mental health? Journal of Developmental and Behavioral Pediatrics, $17,77-83$.

Jaser, S., Whittemore, R., Ambrosino, J., Lindemann, E., \& Grey, M. (2008). Mediators of depressive symptoms in children with type 1 diabetes and their mothers. Journal of Pediatric Psychology, 33, 509-519. doi:10.1093/jpepsy/jsm104.

Kazak, A. (1997). A contextual family/systems approach to pediatric psychology: Introduction to the special issue. Journal of Pediatric Psychology, 22, 141-148. doi: 10.1093/jpepsy/22.2.141

Kovaks, M., Obrosky, D., Goldston, D., \& Bonar, L. (1997). Psychiatric disorders in youths with IDDM: Rates and risk factors. Diabetes Care, 20, 36-44. doi:

10.2337/diacare.20.1.36

Lazarus, R. S. \& Folkman, S. (1984). Stress, appraisal, and coping. New York, NY: Springer.

Lustig, J., Ireys, H., Sills, E., \& Walsh, B. (1996). Mental health of mothers of children with juvenile rheumatoid arthritis: Appraisal as a mediator. Journal of Pediatric Psychology, 21, 719-733. doi: 10.1093/jpepsy/21.5.633

Manuel, J. C. (2001). Risk and resistance factors in the adaptation in mothers of children with juvenile rheumatoid arthritis. Journal of Pediatric Psychology, 26, 237-246. doi: 10.1093/jpepsy/26.4.237

Matos, P.M., \& Fontaine, M. (1992). Family Environment Scale: Adaptação portuguesa [Family Environment Scale: Portuguese version]. Unpublished manuscript, Faculdade de Psicologia e de Ciências da Educação, Universidade do Porto, Porto, Portugal.

Missotten, L., Luyckx, K., \& Seiffge-Krenke, I. (2012). Family climate of adolescents with and without type 1 diabetes: Longitudinal associations with psychosocial adaptation. 
Journal of Child and Family Studies. Advance online publication. doi: 10.1007/s10826012-9585-1

Moos, R.H., \& Moos, B.S. (1986). Family Environment Scale Manual (2nd ed.). Palo Alto, CA: Consulting Psychologists Press.

Mullins, L., Fuemmeler, B., Hoff, A., Chaney, J., Pelt, J., \& Ewing, C. (2004). The relationship of parental overprotection and perceived child vulnerability to depressive symptomatology in children with type 1 diabetes mellitus: The moderating influence of parenting stress. Children's Health Care, 33, 21-34. doi: 10.1207/s15326888chc3301_2

Mullins, L., Olson, R., Reyes, S., Bernardy, N., Huszti, H., \& Volk, R. (1991). Risk and resistance factors in the adaptation of mothers of children with cystic fibrosis. Journal of Pediatric Psychology, 16, 701-715. doi: 10.1093/jpepsy/16.6.701

Overstreet, S., Goins, J., Chen, R., Holmes, S., Greer, T., Dunlap, W., \& Frentz, J. (1995). Family environment and the interrelation of family structure, child behavior, and metabolic control for children with diabetes. Journal of Pediatric Psychology, 20, 435447. doi: 10.1093/jpepsy/20.4.435

Pais-Ribeiro, J., Silva, I., Ferreira, T., Martins, A., Meneses, R., \& Baltar, M. (2007).

Validation study of a portuguese version of the hospital anxiety and depression scale. Psychology, Health \& Medicine, 12, 225-235. doi:10.1080/13548500500524088

Pereira, M., Melo, C., Gameiro, S., \& Canavarro, M. C. (2011). Estudos psicométricos da versão em Português Europeu do índice de qualidade de vida EUROHIS-QOL-8. [Psychometric studies of the European Portuguese version of the quality of life index EUROHIS-QOL-8]. Laboratório de Psicologia, 9, 109-123.

Preacher, K. J., Rucker, D. D., \& Hayes, A. F. (2007). Addressing moderated mediation hypotheses: Theory, Methods, and Prescriptions. Multivariate Behavioral Research, 42, $185-227$. 
Radcliffe, J., Bennett, D., Kazak, A., Foley, B., \& Phillips, P. C. (1996). Adjustment in childhood brain tumor survival: Child, mother, and teacher report. Journal of Pediatric Psychology, 21, 529-539.

Rentinck, I. C. , Ketelaar, M., Jongmans, M. J., \& Gorter, J. W. (2006). Parents of children with cerebral palsy: A review of factors related to the process of adaptation. Child: Care, Health and Development, 33, 161-169.

Schaaijk, N. M., Roeleveld-Versteegh, A. B., \& Baar, A. L. (2013). The interrelationships among paternal and maternal parenting stress, metabolic control, and depressive symptoms in adolescents with Type 1 Diabetes Mellitus. Journal of Pediatric Psychology, $38(1), 30-40$.

Schmidt, S., Mühlan, H., \& Power, M. (2006). The EUROHIS-QOL 8-item index: Psychometric results of a cross-cultural field study. European Journal of Public Health, 16, 420-428. doi:10.1093/eurpub/cki155

Small, S., A., Eastman, G., \& Cornelius, S. (1988). Adolescent autonomy and parental stress. Journal of Youth and Adolescence, 17, 377-391. doi: 10.1007/BF01537880

Snaith, R. P. (2003). The Hospital and Anxiety and Depression Scale. Health and Quality of Life Outcomes, 1, 29. doi:10.1186/1477-7525-1-29

Soliday, E., Kool, E., \& Lande, M. (2001). Family environment, child behavior, and medical indicators in children with kidney disease. Child Psychiatry and Human Development, 31, 279-295. doi: 10.1023/A:1010282305881

Stein, R. K., \& Jessop, D. J. (2003). The Impact on Family Scale revisited: Further psychometric data. Journal of Developmental and Behavioral Pediatrics, 24, 9-16.

Streisand, R., Braniecki, S., Tercyak, K. P., Kazak, A. E. (2001). Childhood illness-related parenting stress: The pediatric inventory for parents. Journal of Pediatric Psychology, 26, 155-162. doi: 10.1093/jpepsy/26.3.155 
Streisand, R., Mackey, E., \& Herge, W. (2010). Associations of parent coping, stress, and well-being in mothers of children with diabetes: Examination of data from a national sample. Maternal and Child Health Journal, 14, 612-617. doi: 10.1007/s10995-009-04977

Streisand, R., Mackey, E., Elliot, B., Mednick, L., Slaughter, I., Turek, J. \& Austin, A. (2008). Parental anxiety and depression associated with caring for a child newly diagnosed with type 1 diabetes: Opportunities for education and counseling. Patient Education and Counseling, 73, 333-338. doi: 10.1016/j.pec.2008.06.014.

Streisand, R., Swift, E., Wickmark, T., Chen, R., \& Holmes, C. (2005). Pediatric parenting stress among parents of children with type 1 diabetes: The role of self-efficacy, responsibility and fear. Journal of Pediatric Psychology, 30, 513-521. doi: 10.1093/jpepsy/jsi076

Tabachnick, B. G., \& Fidell, L. S. (2007). Using multivariate statistics, (5th ed). Boston: Allyn and Bacon.

Wallander, J. L., Thompson, R. J., \& Alriksson-Schmidt, A. (1995). Psychosocial adjustment of children with chronic physical conditions (pp. 141-158). In M. Roberts (Ed.). Handbook of pediatric psychology. New York, NY: The Guilford Press.

Wallander, J. L., \& Varni, J. W. (1992). Adjustment in children with chronic physical disorders: Programmatic research on a disability-stress-coping model. In A. M. LaGreca, L. Siegal, J. L. Wallander, \& C. E. Walker (Eds.). Stress and coping in child health (pp. 279-298). New York, NY: Guilford Press.

Wallander, J. L., \& Varni, J. W. (1998). Effects of pediatric chronic physical disorders on child and family adjustment. Journal of Child Psychology and Psychiatry, 39, 29-46. doi: $10.1111 / 1469-7610.00302$ 
Wallander, J. L., Varni, J. W., Babani, L., DeHaan, C. B., Wilcox, K. T., \& Banis, H. T. (1989). The social environment and the adaptation of mothers of physically handicapped children. Journal of Pediatric Psychology, 14, 371-187. doi:10.1093/jpepsy/14.3.371

Weissberg-Benchell, J., Nansel, T., Holmbeck, G. Chen, R., Anderson, B. Wysocki, T., ... For the Steering Committee of the Family Management of Diabetes Study. (2009). Generic and diabetes-specific parent-child behaviors and quality of life among youth with type 1 diabetes. Journal of Pediatric Psychology, 34, 977-988. doi:

10.1093/jpepsy/jsp003

WHOQOL Group. (1995). The World Health Organization Quality of Life assessment (WHOQOL): Position paper from the World Health Organization. Social Science \& Medicine, 41, 1403-1409.

Williams, L., Laffel, L., \& Hood, K. (2009). Education and Psychological Aspects Diabetesspecific family conflict and psychological distress in paediatric Type 1 diabetes. Diabetic Medicine, 26, 908-914. doi: 10.1111/j.1464-5491.2009.02794.x

Wysocki, T., Buckloh, L., \& Greco, P. (2009). The psychological context of diabetes mellitus in youths. In M. C. Roberts, \& R. G. Steele (Eds.), Handbook of pediatric psychology (4 ${ }^{\text {th }}$ ed, pp. 287-302). New York: The Guilford Press.

Zigmond, A. P., \& Snaith, R. P. (1983). The Hospital and Depression Scale. Acta Psychiatrica Scandinavica, 67, 361-370. doi:10.1111/j.1600-0447.1983.tb09716.x 
Table 1. Descriptive statistics for G1 and G2 parents $(N=246)$

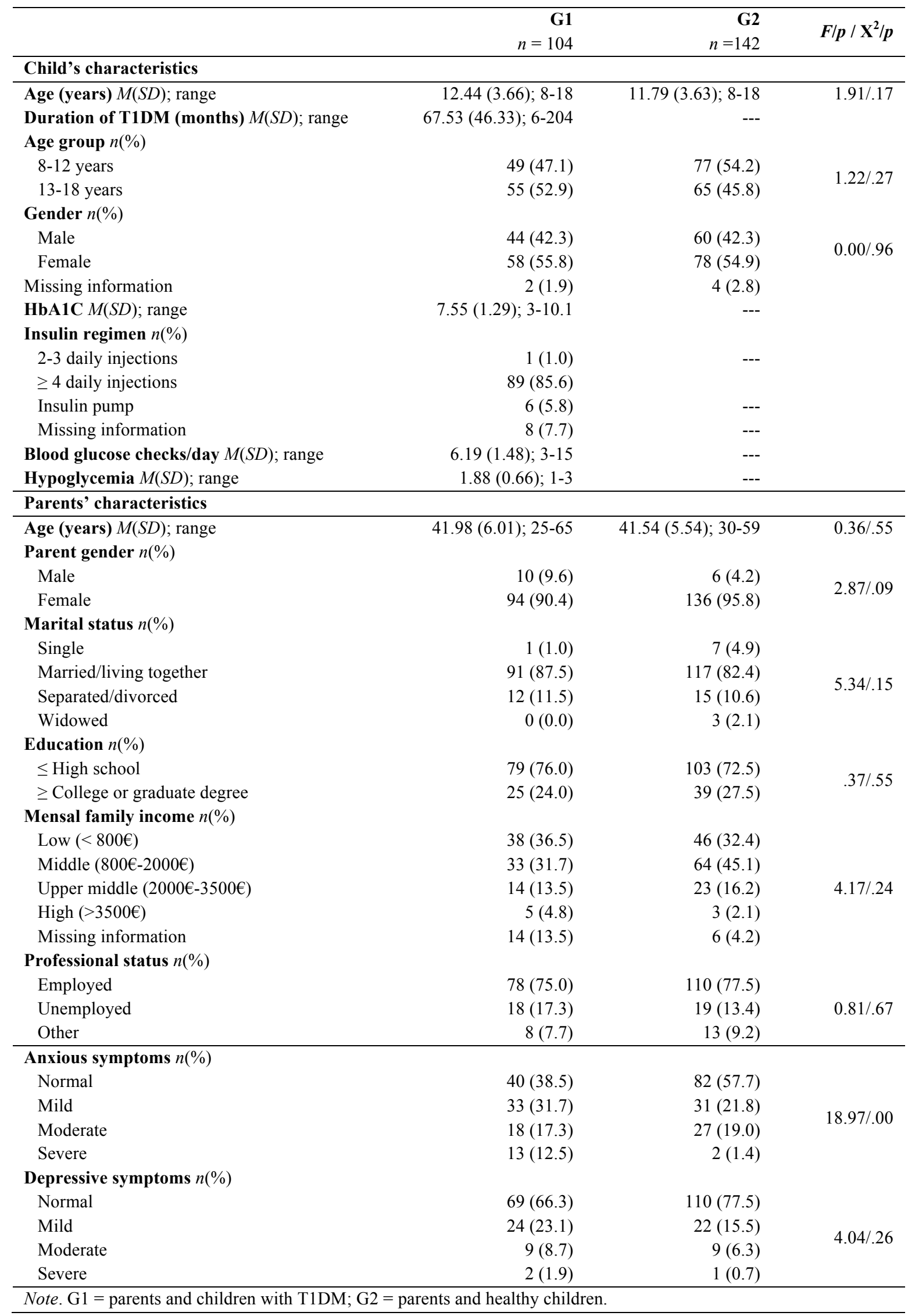


Table 2. Comparisons between G1 and G2 on study variables and matrix of inter-correlations among variables

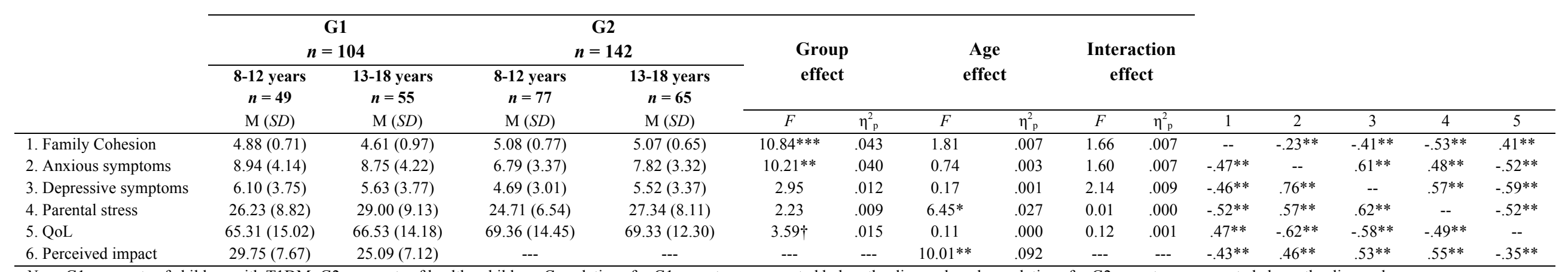

Note. G1 = parents of children with T1DM; G2 = parents of healthy children. Correlations for G1 parents are presented below the diagonal, and correlations for G2 parents are presented above the diagonal.

$\dagger \mathrm{p}<.06 * p<.05 .{ }^{* *} p<.01 .{ }^{* * *} p<.001$. 
Table 3. Summary of simple mediation analyses for models including QoL, parental stress, and depressive and anxious symptomatology (5000 bootstraps).

\begin{tabular}{|c|c|c|c|c|c|c|c|c|}
\hline $\begin{array}{l}\text { Independent } \\
\text { variable }\end{array}$ & $\begin{array}{l}\text { Mediating } \\
\text { variable }\end{array}$ & Dependent variable & $\begin{array}{c}\text { Effect of IV on } \\
\mathrm{M} \\
b(\mathrm{SE})\end{array}$ & $\begin{array}{c}\text { Effect of } M \text { on } \\
\text { DV } \\
b(\mathrm{SE})\end{array}$ & $\begin{array}{c}\text { Direct effect } \\
b(\mathrm{SE})\end{array}$ & \multicolumn{2}{|c|}{$\begin{array}{l}\text { Indirect effect } \\
b \text { (boot SE) }\end{array}$} & $\begin{array}{c}\text { Total effect } \\
b\end{array}$ \\
\hline (IV) & $(\mathrm{M})$ & (DV) & (path $a$ ) & (path $b$ ) & (path $c^{\prime}$ ) & $(a * b)$ & $\begin{array}{c}95 \% \text { CI } \\
\text { (LLCI; ULCI) }\end{array}$ & (path $c$ ) \\
\hline \multirow{3}{*}{$\begin{array}{l}\text { Family } \\
\text { cohesion }\end{array}$} & \multirow{3}{*}{$\begin{array}{l}\text { Perceived } \\
\text { impact }\end{array}$} & QoL & \multirow{3}{*}{$-3.84(0.81)^{* * *}$} & $-0.32(0.18) \dagger$ & $6.99(1.63)^{* * *}$ & $1.24(0.73)$ & $0.06 ; 2.99$ & $8.24 * * *$ \\
\hline & & Parental stress & & $0.48(0.11)^{* * *}$ & $-3.64(0.91)^{* * *}$ & $-1.78(0.57)$ & $-3.13 ;-0.88$ & $-5.42 * * *$ \\
\hline & & Depressive symptoms & & $0.19(0.04)^{* * * *}$ & $-1.27(0.39)^{* *}$ & $-0.76(0.25)$ & $-1.40 ;-0.38$ & $-2.03 * *$ \\
\hline
\end{tabular}

Note. IV = independent variable; $\mathrm{M}=$ mediator; $\mathrm{DV}=$ dependent variable; $\mathrm{SE}=$ standard error; $\mathrm{CI}=$ confidence interval; LLCI = lower limit confidence interval; ULCI $=$ upper limit confidence interval; QoL $=$ quality of life

$\pm \mathrm{t}<.10 * p<.05 . * * p<.01 . * * * p<.001$. 